\title{
Application of a generalized likelihood ratio test statistic to MAGIC data
}

\author{
S. Klepser ${ }^{* \dagger}$, J. Krause ${ }^{* *}$ and J. Sitarek ${ }^{*}$ for the MAGIC Collaboration \\ *IFAE, Edifici Cn., Campus UAB, E-08193 Bellaterra, Spain \\ ${ }^{\dagger}$ Deutsches Elektronen-Synchrotron (DESY), D-15738 Zeuthen, Germany \\ ** Max-Planck-Institut für Physik, D-80805 München, Germany
}

\begin{abstract}
The commonly used detection test statistic for Cherenkov telescope data is Li \& Ma (1983), Eq. 17. It evaluates the compatibility of event counts in an on-source region with those in a representative off-region. It does not exploit the typically known gamma-ray point spread function (PSF) of a system, and in practice its application requires either assumptions on the symmetry of the acceptance across the field of view, or Monte Carlo simulations. MAGIC has an azimuth-dependent, asymmetric acceptance which required a careful review of detection statistics. Besides an adapted Li \& Ma based technique, the recently presented generalized LRT statistic of [1] is now in use. It is more flexible, more sensitive and less systematics-affected, because it is highly customized for multi-pointing Cherenkov telescope data with a known PSF. We present the application of this new method to archival MAGIC data and compare it to the other, Li\&Ma-based method.
\end{abstract}

Keywords: Cherenkov telescopes, test statistic, Li\&Ma

PACS: $95.75 . \mathrm{Pq}$

MAGIC [2] is a Cherenkov telescope system that comprises two large telescopes and cameras with fields of view (FOV) of $3.5^{\circ}$ in diameter. The sensitive trigger area diameters are about $2.0^{\circ}$ and $2.4^{\circ}$. This comparably small area leads to a stereoscopic gamma-ray FOV that is not circularly symmetric and whose shape rotates with the azimuth angle of observation. Uniformity-based background estimation methods like in [3] cannot reliably be applied, neither for skymapping nor source detection in general.

\section{SOURCE DETECTION WITH MAGIC}

What has become the standard way of source detection with MAGIC, at least at low energies, is to observe several positions around the target source coordinate (wobble mode), and apply the so-called Off-from-Wobble-Partner (OfWP) method. In this scheme, the on-source event count of a given wobble set is compared to the off-counts extracted from one or more other wobble sets, using the same focal plane coordinate as the ondata (Fig. 1, left). The off-counts have to be scaled to the on-data, either by effective observation time or background rate. The data of different wobble sets are summed up, and the test statistic of [4], Eq. 17 can be applied, assuming an effective $\alpha$-parameter of $\alpha_{\text {eff }}=\sum_{\omega} \alpha_{\omega}^{2} N_{\omega}^{\text {off }} / \sum_{\omega} \alpha_{\omega} N_{\omega}^{\text {off }}$, where $\alpha_{\omega}$ are the normalization factors of the wobble sets $\omega$. The validity of this method is a plausible approximation if all $\alpha_{\omega}$ are similar, but is uncertain if not. This happens in unbalanced wobble data, which is particularly likely to happen if the data are binned in azimuth and randomly sized portions of events can occur. A second drawback is that in Li \& Ma, Eq. 17, the gamma-ray PSF is not exploited, 

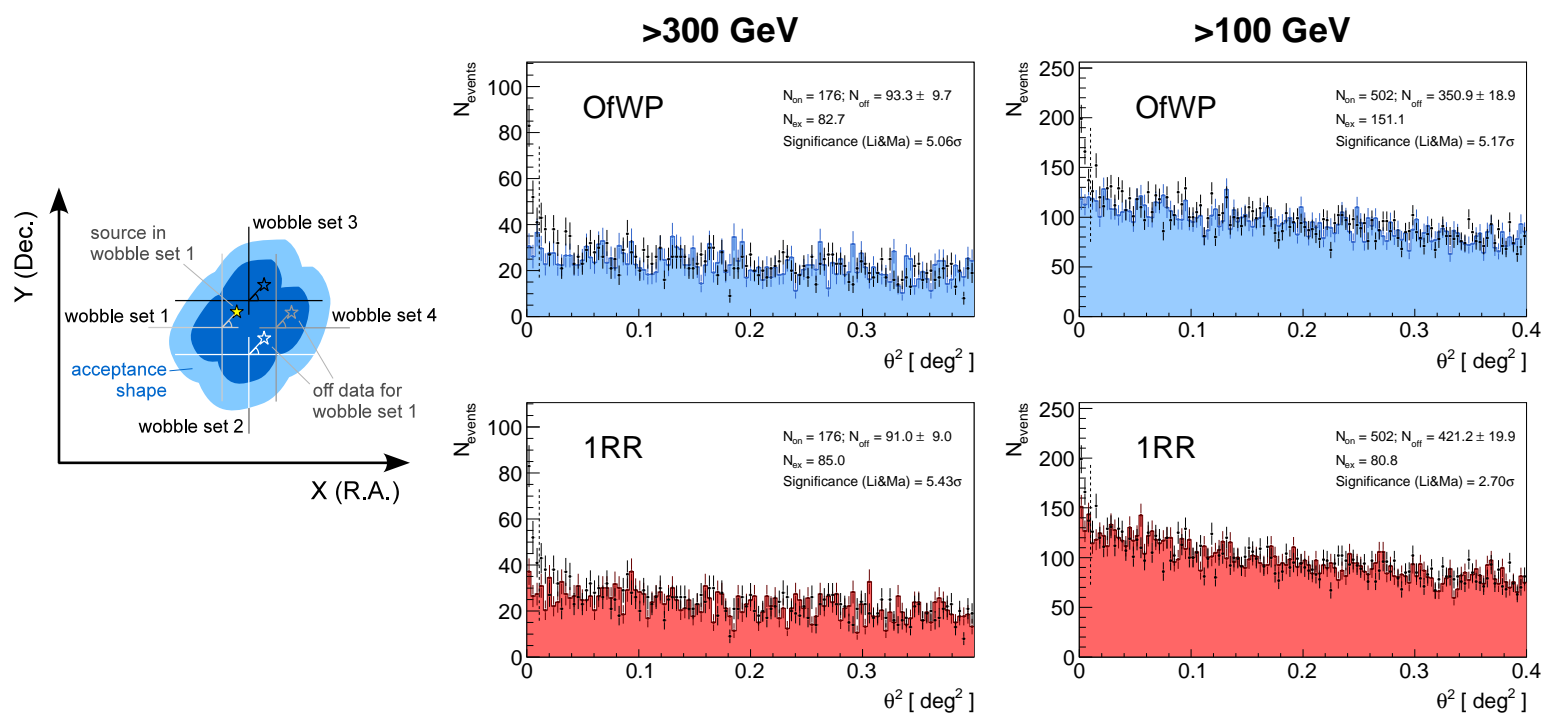

FIGURE 1. Left: The Off-from-Wobble-Partner scheme in a 4-wobble observation. Right: Distributions of squared distance between gamma direction and source position $\left(\theta^{2}\right)$ for high- and low-energy cuts, using OfWP (top row) and 1RR (bottom row) methods, and the Li \& Ma, Eq. 17 test statistic.

although it is a well-known performance parameter that can help to distinguish a signal from a background fluctuation. Finally, the OfWP and "reflected regions" [3] methods are only correct if the off-regions are well-separated from potential sources, and do not overlap with each other. This often requires manual case-by-case consideration, which is not optimal for automatic procedures.

The likelihood ratio test statistic (LRT) of [1], Eq. 16 is a generalized version of the Li \& Ma formula. It is customized to multi-wobble Cherenkov telescope observations. The generalized LRT accommodates that the data are taken in various wobble sets, throughout several "operating conditions" (azimuth or zenith angle, weather, ...), and that a signal, if present, will appear with a possibly known PSF shape. The calculation involves the numerical determination of a relative excess parameter $\phi_{\text {sup }}$, which describes the amplitude of the signal relative to the background.

\section{TEST DATA SET AND STANDARD ANALYSIS}

We use a data subset that comprises two wobble sets in which the radio galaxy IC 310 can marginally be detected at off-axis angles of $0.25^{\circ}$ and $1.0^{\circ}$ from the observed coordinates (see [5] for the physics discussion and analysis of the full data set). We tested the detection significance for two energy cuts $(>100 \mathrm{GeV},>300 \mathrm{GeV})$, using predefined, multi-purpose event selection cuts. On the right side of Fig. 1 is a comparison of detection plots using OfWP and one reflected region (1RR, see [3]). Clearly, OfWP and 1RR are consistent at high energies, but show a significant difference at lower energies. This behaviour is not exceptional, and studies done with off-data show that 
in those cases, OfWP deals better with the inhomogeneous background acceptance and is generally more reliable.

\section{APPLICATION OF THE NEW TEST STATISTIC}

The generalized LRT is implemented in two steps. In the first step, which is part of the MAGIC analysis package MARS [2], the gamma-ray events, on-time and observation coordinates are filled into MARS-independent ROOT ${ }^{1}$ histograms and containers. The data are binned in ranges of azimuth angle to account for the changing acceptance shape. In the second step, the on-source significance and significance skymap are calculated. This latter part uses only ROOT routines and is easily applicable to data of other experiments as well. The whole procedure is automatic, i.e. any number of data sets are combined without any manual effort. The determination of $\phi_{\text {sup }}$ is done using the method of Ridders [6].

\section{Trials and self-consistency check}

The skymaps shown in Fig. 2 are calculated in a fine grid of sky coordinates, leading to correlated significance values. Therefore, the significance distributions are calculated using a much rougher grid with steps of $g=\sqrt{2 \pi} \sigma$, where $\sigma$ is the gamma-ray PSF. This rough scan is repeated 9 times with offsets of $g / 3$, in order not to miss a source. These 9 subscans are conservatively taken into account as independent trials.

Instead of $\theta^{2}$-histograms, the generalized LRT method provides a skymap and significance distribution. This is a much more complete consistency check since the Gaussian nature of the significance value is verified for every detection, which is not possible in a $\theta^{2}$-histogram detection.

\section{RESULTS AND CONCLUSION}

The generalized LRT method was first tested using observations of sky regions without known sources (off-data). No source was found, and the significance distribution was Gaussian as expected. Figure 2 shows the significance skymaps and distributions for high- and low-energy cuts, and also a skymap where the source is incorporated in the null hypothesis (middle column of Fig. 2). The on-source significances are $6.6 \sigma(5.6 \sigma)$ and $6.5 \sigma(5.2 \sigma)$ pre-trial (post-trial), respectively. The significances are somewhat higher than the above OfWP significances, and no systematic inconsistency is found at low energies. The source-including skymap leads to a Gaussian distribution with a post-trial significance of $2.2 \sigma$ - i.e. no additional source is detected.

Concluding, the generalized LRT method [1] was successfully applied to MAGIC data and, as claimed, tends to be more sensitive than the test statistic of Li \& Ma, Eq. 17. It

\footnotetext{
${ }^{1}$ http://root.cern.ch/
} 

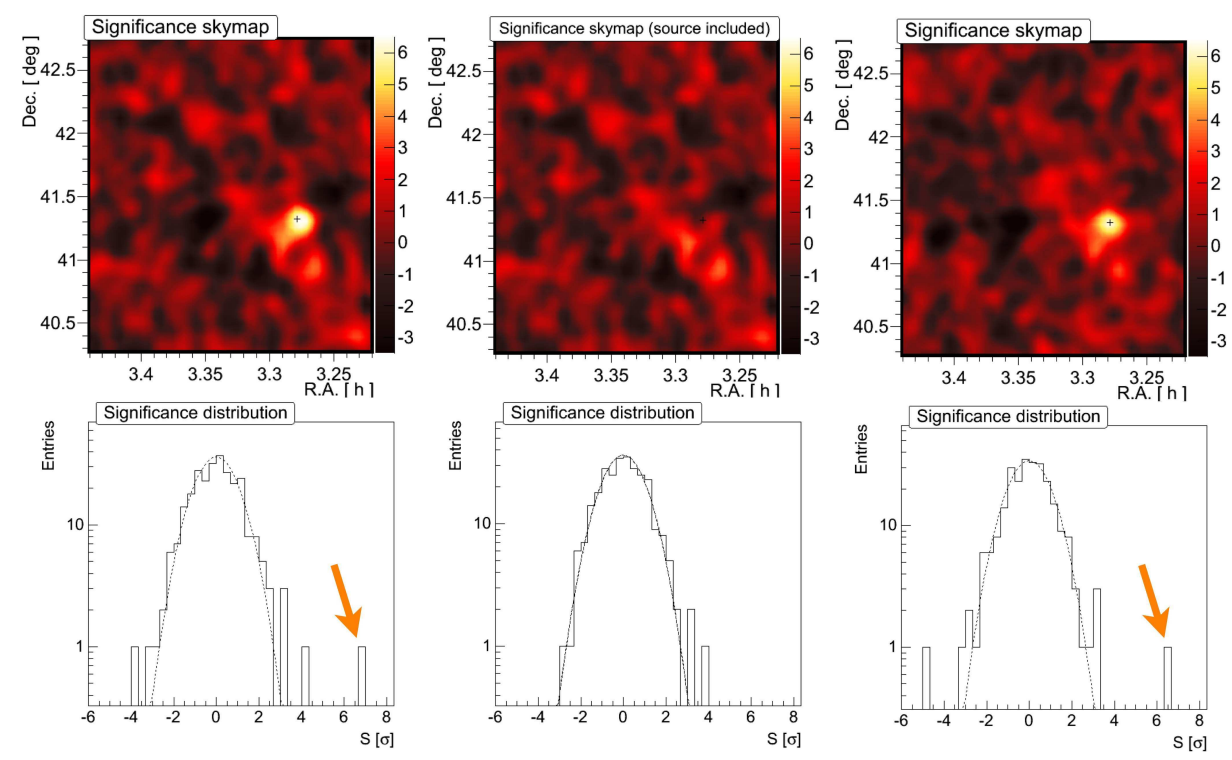

FIGURE 2. Significance skymap and distribution for the high-energy cut ( $>300 \mathrm{GeV}$, left), including the source into the null hypothesis $(>300 \mathrm{GeV}$, middle), and for low energies $(>100 \mathrm{GeV}$, right). The distributions use a rougher sky grid than the skymaps (to avoid correlations, see text). The dotted line is a Gaussian function $(\mu=0, \sigma=1)$.

was implemented as a stand-alone ROOT macro that can be applied flexibly and without manual selection of off-regions.

\section{ACKNOWLEDGMENTS}

We would like to thank the Instituto de Astrofísica de Canarias for the excellent working conditions at the Observatorio del Roque de los Muchachos in La Palma. The support of the German BMBF and MPG, the Italian INFN, the Swiss National Fund SNF, and the Spanish MICINN is gratefully acknowledged. This work was also supported by the CPAN CSD2007-00042 and MultiDark CSD2009-00064 projects of the Spanish Consolider-Ingenio 2010 programme, by grant DO02-353 of the Bulgarian NSF, by grant 127740 of the Academy of Finland, by the DFG Cluster of Excellence "Origin and Structure of the Universe", by the DFG Collaborative Research Centers SFB823/C4 and SFB876/C3, and by the Polish MNiSzW grant 745/N-HESS-MAGIC/2010/0.

\section{REFERENCES}

1. S. Klepser, Astroparticle Physics 36, 64 - 76 (2012), ISSN 0927-6505, arXiv: 1112.0786.

2. J. Aleksić, et al., Astropart. Phys. 35, 435-448 (2012), arXiv: 1108 . 1477.

3. D. Berge, S. Funk, and J. Hinton, A\&A 466, 1219-1229 (2007), a stro-ph/ 0610959.

4. T.-P. Li, and Y.-Q. Ma, ApJ 272, 317-324 (1983).

5. J. Aleksić, et al., ApJL 723, L207-L212 (2010), arXiv: 1009.2155.

6. C. F. J. Ridders, IEEECAS 26, 979-980 (1979). 